\title{
Actin dynamics and the elasticity of cytoskeletal networks
}

\author{
G. A. Buxton ${ }^{*}$, N. Clarke ${ }^{2,3}$, P. J. Hussey ${ }^{2,4}$ \\ ${ }^{1}$ Department of Physics, Case Western Reserve University, Cleveland, OH 44106, USA \\ ${ }^{2}$ Biophysical Sciences Institute, Durham University, Durham, DH1 3LE, UK \\ ${ }^{3}$ Department of Chemistry, Durham University, Durham, DH1 3LE, UK \\ ${ }^{4}$ School of Biological and Biomedical Sciences, Durham University, Durham, DH1 3LE, UK
}

Received 15 April 2009; accepted in revised form 26 June 2009

\begin{abstract}
The structural integrity of a cell depends on its cytoskeleton, which includes an actin network. This network is transient and depends upon the continual polymerization and depolymerization of actin. The degradation of an actin network, and a corresponding reduction in cell stiffness, can indicate the presence of disease. Numerical simulations will be invaluable for understanding the physics of these systems and the correlation between actin dynamics and elasticity. Here we develop a model that is capable of generating actin network structures. In particular, we develop a model of actin dynamics which considers the polymerization, depolymerization, nucleation, severing, and capping of actin filaments. The structures obtained are then fed directly into a mechanical model. This allows us to qualitatively assess the effects of changing various parameters associated with actin dynamics on the elasticity of the material.
\end{abstract}

Keywords: modeling and simulation, biopolymers, actin networks, cytoskeleton

\section{Introduction}

The cytoskeleton is an intricate network of biomacromolecules which pervade the cytoplasm of cells. The structural integrity of a cell depends upon its cytoskeleton, and for small deformations the elasticity of a cell depends on its actin network, a major constituent of the cytoskeleton. Actin networks are dynamic and consist of growing and shrinking filaments, and the cross-linking of these filaments in to a dynamic network. The purpose of this study is to capture the elasticity of actin networks as a function of their dynamic behavior. The correlation between actin dynamics and cellular elasticity can not only reveal interesting insights into the physics of cell mechanics, but also indicate the presence of disease [1]. For example, cancerous cells have been found to exhibit an increase in deformability commensurate with disease progression [2]. Understanding the interplay between cytoskeletal structure and stiffness, therefore, is important for both the diagnosis and treatment of this disease. In this study a computer model of actin dynamics yields network structures which can be directly fed into simulations of network elasticity. In other words, we use computer simulations to directly relate the elastic properties of an actin network to the actin dynamics responsible for the formation of this network.

Living cells have the ability to move and change shape in response to environmental stimuli; a consequence of the dynamic nature of the cytoskeleton and its actin network [3]. In particular, actin networks are formed from actin filaments which are transiently cross-linked by actin-binding proteins (proteins with an affinity for actin). These actin filaments continually polymerize and depolymerize to ensure a recycling of actin monomers, as they treadmill through the filaments, with a net polymer- 
ization at the barbed end typically balancing a net depolymerization at the pointed end [4]. The barbed (+) and pointed (-) ends are structurally different and possess different polymerization kinetics. These dynamics are further regulated by adenosine triphosphate (ATP) hydrolysis; the ATP in free actin monomers that, once polymerized, hydrolyzes to adenosine diphosphate (ADP) and facilitates depolymerization [5]. Furthermore, cellspecific regulatory proteins not only cross-link these dynamic filaments but also cap, sever and help nucleate them [6, 7]. In order to understand the mechanical properties of actin networks we must first elucidate their structure [8]. Therefore, we develop a model which computationally captures these complex dynamics and generates network structures which serve as the input to an elastic model.

There have been many mathematical models of actin polymerization kinetics which yield average quantities [9-11], or simulations of individual filaments [12], however to directly simulate network elasticity, large-scale discrete models are required. For example, Huber et al. [13] have recently presented two-dimensional simulations of actin polymerization which included the diffusion and polymerization of individual actin monomers. Haviv et al. [14] adopted a similar kinetic Monte Carlo approach to model the self-assembly of asters and their transition into stars. Mogilner and Rubinstein [15] have also used stochastic models to simulate actin dynamics during filopedial protrusion. We adopt a similar, but more computationally efficient, approach and consider the filaments to be discretized into $100 \mathrm{~nm}$ segments. Other investigations of actin network elasticity, such as the studies of Huisman et al. [16] or DiDonna and Levine [17], have considered randomly generated network structures.

For many years the physics of elastic networks have attracted attention in relation to the deformation and fracture of heterogeneous materials and structures $[18,19]$. However, recently this emphasis has shifted toward biological networks. Wilhelm and Frey [20] and Head et al. [21] investigated the deformation of two-dimensional networks and found that more densely packed structures deform more affinely (i.e., with a more uniform strain field) and that the individual filaments increasingly deformed more through stretch- ing than through bending. Using a three-dimensional model of network elasticity we recently observed a similar bending-to-stretching transition as a function of network connectivity, which likewise corresponded with a transition to more affine deformations [22]. The ability to computationally capture the mechanics of network structures has led to recent computational investigations of actin networks. Didonna and Levine looked at the effects of protein unfolding in randomly generated twodimensional networks [17]. Huisman et al. [16] looked at the deformation of three-dimensional random network structures as a method of elucidating the mechanics of actin networks. Recently, Broedersz et al. have theoretically looked at networks where the cross-links are assigned stiffnesses much lower than that of the filaments [23]. Here, we expand on these studies by considering network structures which are created directly from a simulation of the underlying actin dynamics. In other words, the output from a model of actin dynamics serves as the input to a mechanical model. The benefits of such a two-step methodology is that the mechanical properties of these networks can be directly correlated with the actin dynamics which led to network formation. We give details of this methodology in the following section, before presenting results and drawing relevant conclusions in subsequent sections.

\section{Methodology}

\subsection{Generation of network}

We employ the first reaction method to simulate actin dynamics, where actin filaments are discretized into regularly spaced nodes along the filament path [24]. Dynamic events are assigned rates at which they are likely to occur; for example, the polymerization and depolymerization at both filament ends, the nucleation of a filament, or the severing, capping and uncapping of existing filaments. Upon capping the polymerization kinetics at the barbed end are inhibited. Polymerization rates are taken to be proportional to the availability of free actin (i.e., $w_{+}=k_{+} \cdot C$, where $C$ is the actin concentration). Nucleation can occur either spontaneously (through the formation of relatively stable trimers) leading to a concentration dependence of the form $w_{\text {new }}=k_{\text {new }} \cdot C^{3}$ [25], or nucleation can be initiated by actin binding proteins, with a rate given by 
$w_{n e w}=k_{\text {new }} \cdot C$. The concentration of free actin is depleted as filaments are created or polymerize, and increased when filaments depolymerize. Due to the high diffusion coefficient of actin, and relatively slow growth of filaments, we assume spatial variations of actin can be neglected. The rate at which a filament is likely to be severed is proportional to its length (i.e., $w_{\text {sever }}=k_{\text {sever }} l$ ). Although it is worth noting that the hydrolysis of ATP-actin may effect the severing rate. For computational efficiency we do not include ATP hydrolysis as one of the kinetic events in the first reaction method, but rather assign a probability of hydrolysis occurring during the time step $\Delta t$ of the form $P_{\text {hydro }}=$ $1-\exp \left(-k_{h y d r o} \cdot \Delta t\right)$, where khydro is the rate of ATP hydrolysis on the actin filament. A filament segment with ATP-actin undergoes hydrolysis if a random number between zero and one is less than $P_{\text {hydro }}$. Upon polymerization a new node at the end of a filament is created with a position $r_{i+1}=$ $r_{i}+r_{0}\left(r_{i}-r_{i-1}+\delta\right) /\left(\left|r_{i}-r_{i-1}+\delta\right|\right)$, where $r_{i+1}$ is the position of the new node, $r_{0}=100 \mathrm{~nm}$ is the spatial discretization of the filament and $\delta$ is a spherically symmetric random vector whose magnitude is chosen to coincide with the persistence length of actin filaments. In particular, the orientational correlation function $<\cos \theta>=\exp \left(-r_{0} / l_{p}\right)$, where $l_{p}=$ $17 \mu \mathrm{m}$ is the persistence length [26, 27]. The rate equations, therefore, do not describe the addition of actual molecular subunits but, rather, the sequential addition of several actin subunits $100 \mathrm{~nm}$ in length. Periodic boundary conditions are enforced on a system of size $L^{3}=(20 \mu \mathrm{m})^{3}$ and filament lengths are limited to $20 \mu \mathrm{m}$ and, therefore, do not span the length of the simulation box; we limit the size of the filaments to ensure that the elasticity of the network is due to the cross-linked network structure and not as a consequence of a single filament spanning the system. While this simulation size is comparable to the average filament length found in this study (typically less than $10 \mu \mathrm{m}$ ), it is still adequate to determine the constitutive response of these systems. In particular, Ostoja-Starzewski and Stahl [28] have found that displacement-based boundary conditions (as used here) can accurately capture the elastic properties of random networks even for relatively small system sizes.

A time is associated with each event of the form $\tau_{i}=-\ln (\varepsilon[0: 1]) / w_{i}$, where wi is the rate of the $i^{\text {th }}$ event and $\varepsilon[0: 1]$ is a random number between
0 and 1 [24]. The event with the lowest $\tau_{i}$ is executed at each iteration and this time is removed from the times associated with the remaining events. New $\tau_{i}$ 's, for the recently performed (and any new) events, are calculated and, again, the event with the lowest $\tau_{i}$ is executed. The process is reiterated until steady state occurs. We start from an initial condition consisting of one hundred $1 \mu \mathrm{m}$ long filaments, however, the final steady-state structures have evolved sufficiently that they are not sensitive to this choice. We run the simulations for $1 \cdot 10^{8}$ iterations (time steps are on the order of milliseconds). The systems considered here are observed to reach steady state before $1 \cdot 10^{7}$ iterations (defined as when the average quantities describing the system reach relatively constant values). Once the steady-state filament structures are established we determine a probability of crosslinking between neighboring segments.

From the tube model of actin filaments the fluctuations of a filament, perpendicular to its length, are believed to be limited to a distance of $400 \mathrm{~nm}$ [29]. Therefore, if the separation distance between two segments is greater than this distance we assume the probability of cross-linking to be zero. If distances are less than $400 \mathrm{~nm}$ the probability of crosslinking is taken to be of the form $P_{\text {cross }}=$ $1-\exp \left(-k_{\text {cross }} \cdot \Delta t\right)$, where $\Delta t$ refers to the time the segments have coexisted and $k_{\text {cross }}$ is a rate of crosslinking. We take $k_{\text {cross }}=k_{A B} \cdot P_{A B}$, where $P_{A B}$ is the probability of contact between two sites, defined as when two filaments (confined within tubes of $400 \mathrm{~nm}$ diameters) come within $40 \mathrm{~nm}$ of each other (the size of a cross-linking protein [30]). Therefore, the probability of contact is simply $P_{A B}=0.01$ and we can vary $k_{A B}$ to generate crosslinked structures with variable cross-link density.

\subsection{Network elasticity}

Once the network structures are obtained we can feed these structures directly into an elastic model and correlate network geometry with elastic properties. The elastic energy of a filament is given by Equation (1) [31]:

$$
A=\frac{1}{2} E a \int\left(\frac{\delta u}{\delta s}\right)^{2} \delta s+\frac{1}{2} E I \int\left(\frac{1}{R}\right)^{2} \delta s
$$

where the first term accounts for filament stretching and the second term filament bending. $E$ is the 
Young's modulus, $a$ is the cross-sectional area, $I$ is the area moment of inertia, $R$ is the radius of curvature and $u$ is the displacement along the filament curvature, $s$. Similar elastic models have proven to be highly successful at simulating the complex deformations of actin networks [16, 17, 32], and elastic networks in general $[20,21]$.

We take the flexural rigidity of the actin filaments to be $E I=7.3 \cdot 10^{-26} \mathrm{~N} \cdot \mathrm{m}^{2}$ [33] and the filament stiffness to be $34.5 \cdot 10^{-3} \mathrm{~N} \cdot \mathrm{m}^{-1}$ [34]. Cross-linking proteins are described by linear springs with a stiffness much lower than that of the actin filaments. In particular, the stiffness is taken to be $5 \cdot 10^{-5}$ [35] and the equilibrium length is taken to be $40 \mathrm{~nm}$ [30]. However, other more complicated elements could be considered; for example, DiDonna and Levine [17] have considered unfolding cross-links which reversibly unfold at a critical pulling force.

Maintaining periodic boundary conditions we can evolve the network to equilibrium using the following Langevin Equation (2):

$$
F+\eta=\xi \frac{\mathrm{d} r}{\mathrm{~d} t}
$$

where $\xi$ is the friction coefficient and $\eta$ is a Gaussian noise term which satisfies the fluctuation dissipation theorem [36]. The filament is discretized into a series of points, or nodes, $100 \mathrm{~nm}$ apart. The velocity of a node is $\mathrm{d} r / \mathrm{d} t$ and the elastic forces acting on this node is $F$. For numerical stability, $\xi / \Delta t$ is taken to be $10 \cdot 10^{-6} \mathrm{~N} \cdot \mathrm{m}^{-1}$ and $\eta=\sqrt{2 k_{b} T \xi / \Delta t} \cdot \mathrm{C}$ where $\Delta t$ is the time step, $k_{b}$ is the Boltzmann constant, $T=300 \mathrm{~K}$ is the temperature, and $G$ is a Gaussian distributed random number with zero mean and unit standard deviation. Incorporating thermal noise into the relaxation dynamics ensures that we capture entropic effects. The shear modulus can then be obtained from the elastic energy density stored in a network as a consequence of the applied shear. This requires us to contrast the elastic energy in a given network subject to an applied shear (using Lee-Edwards boundary conditions) with the elastic energy in the same network without applied shear (but still in a non-equilibrium state due to thermal noise). In other words, the difference in elastic energy is related to shear modulus by $A_{\text {shear }}-A_{\text {undeform }}=G \cdot \gamma^{2} / 2$, where $A_{\text {shear }}$ and $A_{\text {undeform }}$ are the elastic energy densities in the sheared and undeformed networks, respectively [31]. Note that the elastic energy density is the total energy stored within all the elastic filaments in the simulation, divided by the volume of the simulation. $G$ is the shear modulus and $\gamma$ is the applied shear. The stretching and bending of actin filaments, or stretching of cross-link proteins, results in forces acting on the discrete nodes which characterize the filament backbone. These forces are used to evolve the Langevin equation, until the system relaxes to equilibrium. The difference in elastic energy density between equilibrated systems with and without applied shear can then be used to calculate the shear modulus. Furthermore, not only can we calculate the shear modulus but also isolate the contribution from different structural elements (stretching and bending energy of actin filaments and stretching energy of cross-linking proteins). The averages and standard deviations are presented from three independent simulation (with each simulation taking approximately 100 hours of cpu time on a standard linux processor).

\section{Results and discussion}

The polymerization kinetics are taken from Pollard et al. [7] (Table 1.). Note that due to our discretization the polymerization rates used in the simulation differ from those quoted and correspond to the polymerization of $100 \mathrm{~nm}$ pieces, rather than molecular subunits. The rate of hydrolysis is taken to be $0.3 \mathrm{~s}^{-1}$ [37] and the capping rate at the barbed end is taken to be $1 \mathrm{~s}^{-1}$ (although this will depend on the concentration of capping proteins). We vary the nucleation rate (for systems where the nucleation rate is either proportional to concentration, or the concentration cubed), the severing rate, the uncapping rate and the concentration of actin. The range of these parameters are given in Table 2 .

Figure 1 shows a snapshot of a simulation with $k_{\text {new }}=0.1 \mu \mathrm{M}^{-1} \cdot \mathrm{s}^{-1} \cdot \mu \mathrm{m}^{-3}, k_{\text {sever }}=1 \cdot 10^{-6} \mathrm{~s}^{-1} \cdot \mu \mathrm{m}^{-1}$, $k_{\text {uncap }}=0.1 \mathrm{~s}^{-1}$ and $\theta=1 \mu \mathrm{M}$. The network is connected with a rate of cross-linking $k_{A B}=1 \mathrm{~s}^{-1}$. The

Table 1. Polymerization kinetics [7]

\begin{tabular}{|l|l|}
\hline Polymerization at barbed end with ATP-actin & $11.6 \mathrm{~s}^{-1} \mu \mathrm{M}^{-1}$ \\
\hline Depolymerization at barbed end with ATP-actin & $1.4 \mathrm{~s}^{-1}$ \\
\hline Polymerization at pointed end with ADP-actin & $0.16 \mathrm{~s}^{-1} \mu \mathrm{M}^{-1}$ \\
\hline Depolymerization at pointed end with ADP-actin & $0.27 \mathrm{~s}^{-1}$ \\
\hline Polymerization at barbed end with ADP-actin & $3.8 \mathrm{~s}^{-1} \mu \mathrm{M}^{-1}$ \\
\hline Depolymerization at barbed end with ADP-actin & $7.2 \mathrm{~s}^{-1}$ \\
\hline Polymerization at pointed end with ATP-actin & $1.3 \mathrm{~s}^{-1} \mu \mathrm{M}^{-1}$ \\
\hline Depolymerization at pointed end with ATP-actin & $0.8 \mathrm{~s}^{-1}$ \\
\hline
\end{tabular}


Table 2. Polymerization rates

\begin{tabular}{|l|l|}
\hline Nucleation rate & $k_{\text {new }}=0.1-10 \mu \mathrm{M}^{-1} \cdot \mathrm{s}^{-1} \cdot \mu \mathrm{m}^{-3}$ \\
\hline Severing rate & $k_{\text {sever }}=1 \cdot 10^{-8}-1 \cdot 10^{-4} \mathrm{~s}^{-1} \cdot \mu \mathrm{m}^{-1}$ \\
\hline Uncapping rate & $k_{\text {uncap }}=0-1 \mathrm{~s}^{-1}$ \\
\hline Actin concentration & $\theta=0.5-1.5 \mu \mathrm{M}$ \\
\hline
\end{tabular}

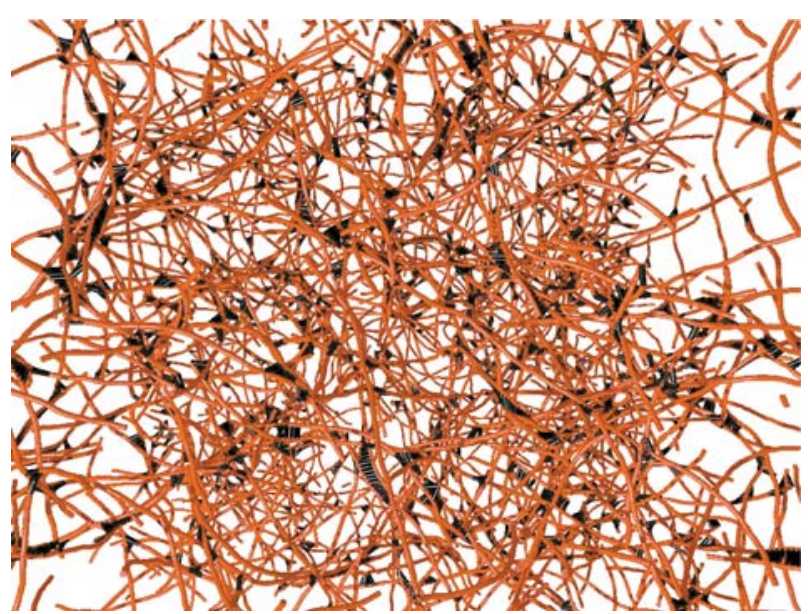

Figure 1. Snapshot of a simulation with $k_{\text {new }}=$ $0.1 \mu \mathrm{M}^{-1} \cdot \mathrm{s}^{-1} \cdot \mu \mathrm{m}^{-3}, k_{\text {sever }}=1 \cdot 10^{-6} \mathrm{~s}^{-1} \cdot \mu \mathrm{m}^{-1}$, $k_{\text {uncap }}=0.1 \mathrm{~s}^{-1}$ and $\theta=1 \mu \mathrm{M}$. The network is connected with a rate of cross-linking $k_{A B}=$ $1 \mathrm{~s}^{-1}$. The filaments are shown as red cylinders and the cross-linking proteins are shown as black cylinders.

filaments are shown as red cylinders and the crosslinking proteins are shown as black cylinders. For this value of $k_{A B}$ we find $6320 \pm 8$ cross-linking proteins (connecting 609 filaments with an average length of $8.2 \mu \mathrm{m})$. For smaller values of $k_{A B}=0.01$ and 0.1 , we find $2304 \pm 39$ and $5568 \pm 25$ proteins, respectively. In this study we maintain $k_{A B}=1$ which corresponds to a relatively high cross-link density and note that smaller values of $k_{A B}$ will increase the compliance of the networks.

The effects of varying the nucleation rate is shown in Figure 2. We vary the nucleation rate from 0.1 to $10 \mu \mathrm{M}^{-1} \cdot \mathrm{s}^{-1} \cdot \mu \mathrm{m}^{-3}$ (or $\mu \mathrm{M}^{-3} \cdot \mathrm{s}^{-1} \cdot \mu \mathrm{m}^{-3}$ for $w_{\text {new }}=$ $k_{n e w} \cdot C^{3}$ ). We contrast this difference in power and depict the average length of the filaments and the number of filaments. For power $=1\left(w_{\text {new }}=k_{\text {new }} \cdot C\right)$ we find the average number of filaments increases from 500 to 3500 and the length decreases from 9 to $2 \mu \mathrm{m}$. For power $=3\left(w_{\text {new }}=k_{\text {new }} \cdot C^{3}\right)$ the effects of varying $k_{\text {new }}$ are less severe. The concentration of free actin in the systems considered here is roughly $0.6 \mu \mathrm{m}$ and so the effects of increasing $k_{n e w}$ are expected to be 3 times less for systems where power $=3$.

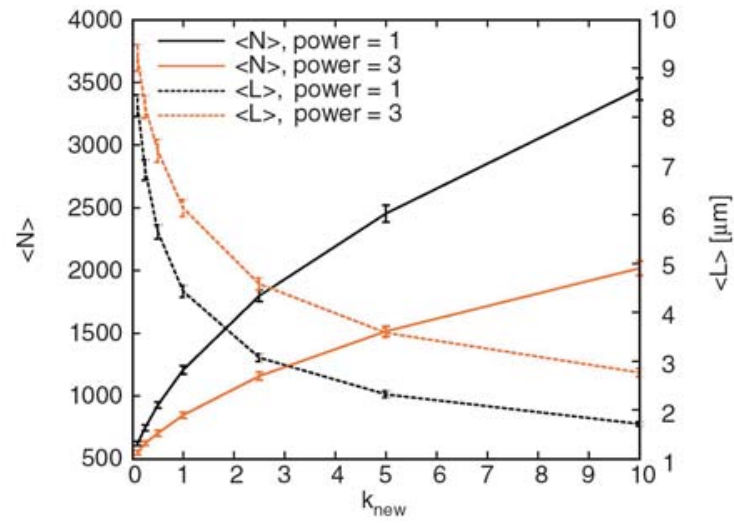

Figure 2. The effects of varying the nucleation rate from 0.1 to $10 \mu \mathrm{M}^{-1} \cdot \mathrm{s}^{-1} \cdot \mu \mathrm{m}^{-3}$ (or $\mu \mathrm{M}^{-3} \cdot \mathrm{s}^{-1} \cdot \mu \mathrm{m}^{-3}$ for $w_{\text {new }}=k_{\text {new }} \cdot \mathrm{C}^{3}$ ) on the number and average length of actin filaments. $k_{\text {sever }}=1 \cdot 10^{-6} \mathrm{~s}^{-1} \cdot \mu \mathrm{m}^{-1}$, $k_{\text {uncap }}=0.1 \mathrm{~s}^{-1}$ and $\theta=1 \mu \mathrm{M}$. Nucleation rates of the form $w_{\text {new }}=k_{\text {new }} \cdot C$ are contrasted with nucleation rates of the form $w_{\text {new }}=k_{\text {new }} \cdot C^{3}$ to compare spontaneous with protein mediated nucleation.

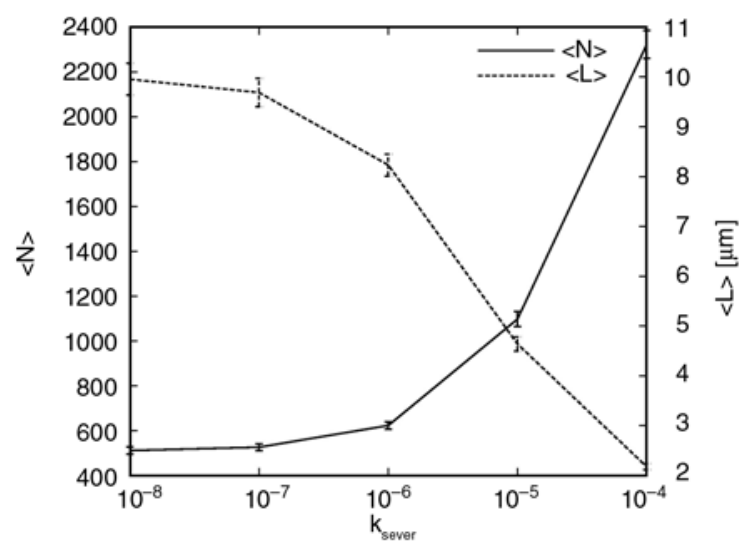

Figure 3. The effects of varying the severing rate over orders of magnitude (from $1 \cdot 10^{-8}$ to $1 \cdot 10^{-4}$ ) on the number and average length of actin filaments. $k_{\text {new }}=0.1 \mu \mathrm{M}^{-1} \cdot \mathrm{s}^{-1} \cdot \mu \mathrm{m}^{-3}, k_{\text {uncap }}=0.1 \mathrm{~s}^{-1}$ and $\theta=1 \mu \mathrm{M}$

Figure 3 shows the effects of varying $k_{\text {sever }}$ from $1 \cdot 10^{-8}$ to $1 \cdot 10^{-4}$. As expected the number of filaments increases and the average length decrease. In particular, the number of filaments increases from 500 to 2200 and the average length decreases from 10 to $2 \mu \mathrm{m}$. This range of filament lengths is comparable to those found in experimental studies [38]. Figure 4 shows the effects of increasing the uncapping rate from 0.001 to $1 \mathrm{~s}^{-1}$. The number of filaments initially decreases before increasing, while the average filament length doubles from 6 to $12 \mu \mathrm{m}$. The percentage of filaments that are capped as a function of uncapping rate is shown in the inset. As filaments become increasingly uncapped 


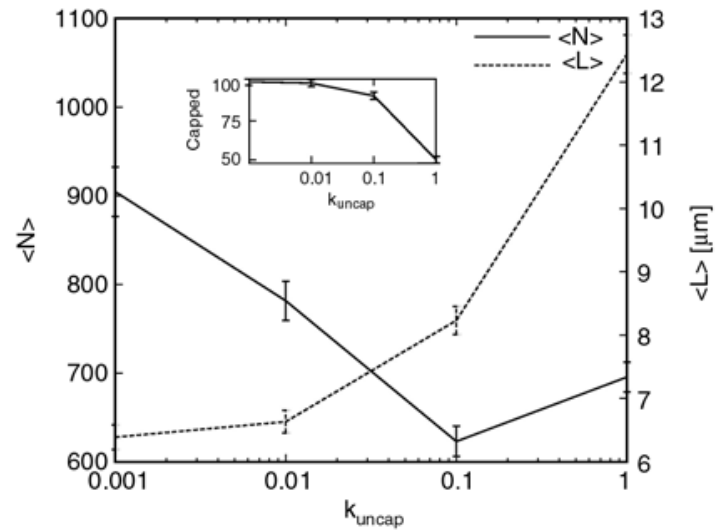

Figure 4. The effects of varying the uncapping rate (at the barbed end) from 0.001 to $1 \mathrm{~s}^{-1}$ on the number and average length of actin filaments. Note the capping rate is kept at $1 \mathrm{~s}^{-1} \cdot k_{\text {new }}=$ $0.1 \mu \mathrm{M}^{-1} \cdot \mathrm{s}^{-1} \cdot \mu \mathrm{m}^{-3}, k_{\text {sever }}=1 \cdot 10^{-6} \mathrm{~s}^{-1} \cdot \mu \mathrm{m}^{-1}$ and $\theta=1 \mu \mathrm{M}$. The percentage of filaments which are capped is shown within the inset.

the length increases, as the polymerization kinetics are less inhibited, and the reduction in free actin reduces the nucleation rate. However, newly created filaments have a greater chance of survival as they are less likely to be capped. This reduction in filament nucleation, but increasing likelihood of filament survival, results in the number of filaments initially decreasing before increasing.

The effects of increasing actin concentration are shown in Figure 5. For an actin concentration of $0.5 \mu \mathrm{M}$ there are relatively few filaments of very small length. As the concentration is increased to $1.5 \mu \mathrm{M}$ the number of filaments increases linearly to 1200 and the average filament length increases to $9 \mu \mathrm{m}$. Newly created filaments consist of ATP-

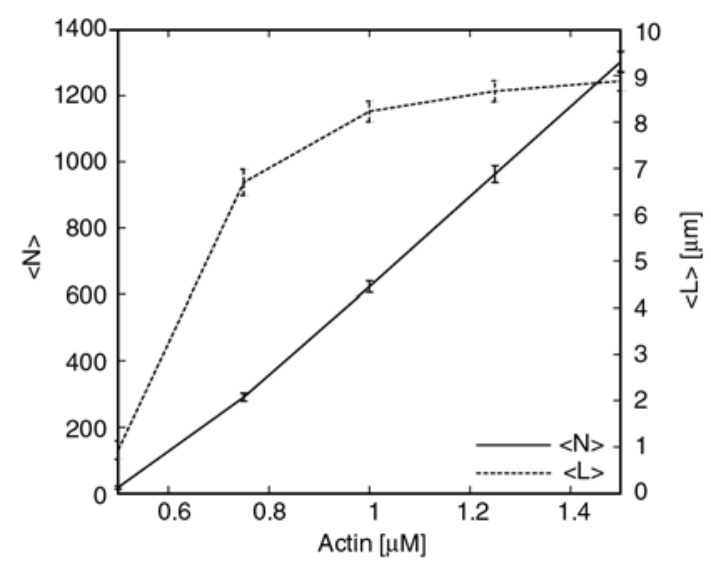

Figure 5. The effects of varying the total actin concentration from 0.5 to $1.5 \mu \mathrm{M}$ on the number and average length of actin filaments. $k_{\text {new }}=$ $0.1 \mu \mathrm{M}^{-1} \cdot \mathrm{s}^{-1} \cdot \mu \mathrm{m}^{-3}, k_{\text {sever }}=1 \cdot 10^{-6} \mathrm{~s}^{-1} \cdot \mu \mathrm{m}^{-1}$ and $k_{\text {uncap }}=0.1 \mathrm{~s}^{-1}$ actin and the critical concentration at the pointed end with ATP-actin is 0.61 . Therefore, the newly created filaments with concentrations less than this depolymerize from the pointed end and have less chance of survival, whereas for concentrations greater than 0.61 the filaments initially grow from both ends before hydrolysis ensures the preferential depolymerization of the pointed end. We, therefore, see a dramatic increase in filament length around a concentration of 0.61 .

We now turn our attention to the mechanical properties of these networks. Figure 6 shows the effects of varying actin concentration on the shear modulus of the network. For the simulations shown in Figure 5 we feed the structures in to the mechanical model and calculate the shear modulus. The error bars correspond to the standard deviation of three runs. In particular, we plot the shear modulus as a function of F-actin concentration. As the total concentration of actin is increased to $1.5 \mu \mathrm{M}$ the concentration of F-actin increases to $0.9 \mu \mathrm{M}$. This results in an exponential increase in the mechanical stiffness of the material. The shear modulus increases to $7 \mathrm{~Pa}$ as not only the density of the filaments increases, but also the number of cross-linking proteins increases. This range of shear modulus is consistent with experimental studies [39-41] although the shear modulus varies as the concentration to the power of 4.7 (rather than 2.5 as predicted theoretically and found in Gardel et al. [41]). This might be a consequence of the elastic energy being primarily stored within the cross-linking proteins and not the actin filaments.

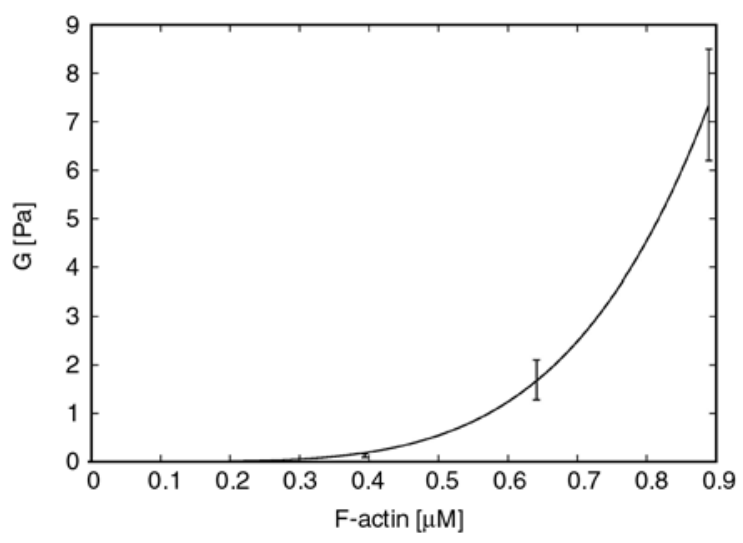

Figure 6. Plot of shear modulus, $G$, as a function of Factin concentration. The variation in $\mathrm{F}$-actin is obtained through varying the total actin concentration (see Figure 5) 


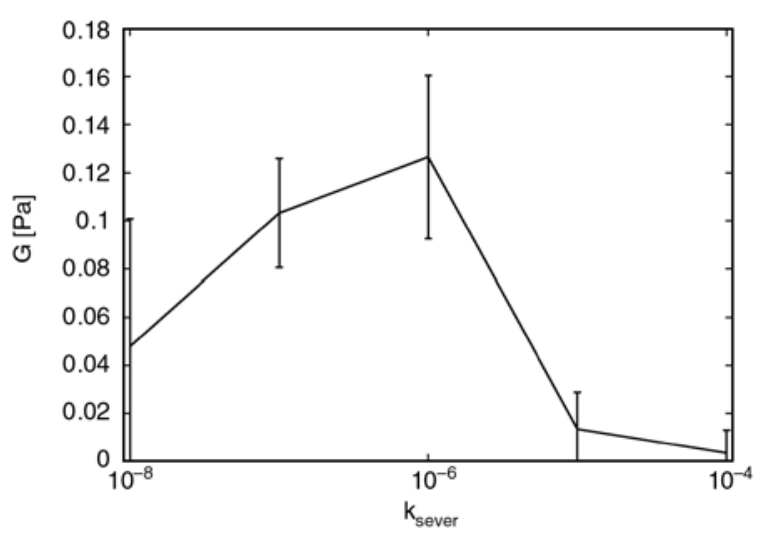

Figure 7. Plot of shear modulus as a function of severing rate. Increasing severing rates corresponds with a change in network structure from networks consisting of less larger filaments to more smaller filaments (see Figure 3)

The effects of increasing the severing rate in Figure 3 predictably resulted in an increase in the number of the filaments and a reduction in filament length. Figure 7 shows the effect of this on the mechanical properties of the actin networks. While the concentration of F-actin also shows a slight variation as a function of severing rate (not shown) the variations are on the order of a percent. The variation in mechanical stiffness is, therefore, a consequence of network geometry. In particular, neither the networks consisting of many small filaments, nor the networks consisting of just a few hundred large filaments, appear to provide the best mechanical properties. Interestingly, the optimum mechanical performance occurred in systems in between these two extremes.

\section{Conclusions}

To summarize, we have coupled a model of actin dynamics with a mechanical model to directly correlate network formation and elasticity. We model the network formation through a model which considers the rates at which various events occur (polymerization, depolymerization, nucleation, severing, capping, uncapping and hydrolysis). This is then fed directly into an elastic model which allows us to obtain a shear modulus for the structure. These preliminary results offer interesting physical insights into these systems, and provide a platform for developing specific biological models which could be directly compared to commensurate experimental studies.
Future work will further explore the parameter space and analyze the statistics of these heterogeneous networks. In particular, the severing rate might depend on the hydrolysis of ATP-actin and vary along the length of a filament (with ADP-actin more likely to sever [10]) or the heterogeneity of actin concentration could be important in modeling more realistic cellular environments. In terms of the mechanical simulations, the incorporation of nonlinear protein deformations as a consequence of protein unfolding could improve the dynamics of the model and future work could explore the effects of varying cross-link density.

The focus in the current study, however, was the development of a two-step methodology capable of correlating actin dynamics with actin network mechanics. Our new methodology could provide insights into how variations in actin dynamics between different systems, or in response to a disease [2], can ultimately effect the mechanical properties of the cell.

\section{Acknowledgements}

We thank Dr Junli Liu and Dr Bernard Piette (Durham University) for their critical reading of the manuscript, and the referees for their useful and insightful comments.

\section{References}

[1] Costa K. D.: Single-cell elastography: Probing for disease with the atomic force microscope. Disease Markers, 19, 139-154 (2003).

[2] Guck J., Schinkinger S., Lincoln B., Wottawah F., Ebert S., Romeyke M., Lenz D., Erickson H. M., Ananthakrishnan R., Mitchell D., Kas J., Ulvick S., Bilby C.: Optical deformability as an inherent cell marker for testing malignant transformation and metastatic competence. Biophysical Journal, 88, 3689-3698 (2005). DOI: 10.1529/biophysj.104.045476

[3] Alberts B., Bray D., Johnson A., Lewis J., Raff M., Roberts K., Walter P.: Essential cell biology. An introduction to the molecular biology of the cell. Garland Publishing, New York (1998).

[4] Fujiwara I., Takahashi S., Tadakuma H., Funatsu T., Ishiwata S.: Microscopic analysis of polymerization dynamics with individual actin filaments. Nature Cell Biology, 4, 666-673 (2002). DOI: $10.1038 / \mathrm{ncb} 841$

[5] Korn E. D., Carlier M., Pantaloni D.: Actin polymerization and ATP hydrolysis. Science, 238, 638-644 (1987).

DOI: $10.1126 /$ science. 3672117 
[6] Pollard T. D., Cooper J. A.: Actin and actin-binding proteins. A critical evaluation of mechanisms and functions. Annual Review of Biochemistry, 55, 9871035 (1986).

DOI: 10.1146/annurev.bi.55.070186.005011

[7] Pollard T. D., Blanchoin L., Mullins R. D.: Molecular mechanisms controlling actin filament dynamics in nonmuscle cells. Annual Review of Biophysics and Biomolecular Structure, 29, 545-576 (2000).

DOI: $10.1146 /$ annurev.biophys.29.1.545

[8] Nossal R.: On the elasticity of cytoskeletal networks. Biophysical Journal, 53, 349-359 (1988). DOI: 10.1016/S0006-3495(88)83112-6

[9] Ermentrout G. B., Edelstein-Keshet L.: Models for the length distributions of actin filaments II: Polymerization and fragmentation by gelsolin acting together. Bulletin of Mathematical Biology, 60, 477-503 (1998). DOI: $10.1006 /$ bulm.1997.0012

[10] Edelstein-Keshet L., Ermentrout G. B.: A model of actin filament length distribution in a lamellipod. Journal of Mathematical Biology, 43, 325-355 (2001). DOI: $10.1007 / \mathrm{s} 002850100102$

[11] Pollard T. D., Berro J.: Mathematical models and simulations of cellular processes based on actin filaments. Journal of Biological Chemistry, 284, 5433-5437 (2009). DOI: $10.1074 / \mathrm{jbc} . \mathrm{R} 800043200$

[12] Roland J., Berro J., Michelot A., Blanchion L., Martiel J-L.: Stochastic severing of actin filaments by actin depolymerizing factor/cofilin controls the emergence of a steady dynamical regime. Biophysical Journal, 94, 2082-2094 (2008). DOI: $10.1529 /$ biophysj.107.121988

[13] Huber F., Käs J., Stuhrmann B.: Growing actin networks from lamellipodium and lamellum by selfassembly. Biophysical Journal, 95, 5508-5523 (2008). DOI: $10.1529 /$ biophysj.108.134817

[14] Haviv L., Brill-Karniely Y., Mahaffy R., Backouche F., Ben-Shaul A., Pollard T. D., BernheimGroswasser A.: Reconstitution of the transition from lamellipodium to filopodium in a membrane-free system. Proceedings of the National Academy Sciences, 103, 4906-4911 (2006).

DOI: $10.1073 /$ pnas.0508269103

[15] Mogilner A., Rubinstein B.: The physics of filopodial protrusion. Biophysical Journal, 89, 782-795 (2005). DOI: $10.1529 /$ biophysj.104.056515

[16] Huisman E. M., van Dillen T., Onck P. R., van der Giessen E.: Three-dimensional cross-linked F-actin networks: Relation between network architecture and mechanical behavior. Physical Review Letters, 99, 208103/1-208103/4 (2007). DOI: 10.1103/PhysRevLett.99.208103

[17] DiDonna B. A., Levine A.: Filamin cross-linked semiflexible networks: Fragility under strain. Physical Review Letters, 97, 068104/1-068104/4 (2006). DOI: 10.1103/PhysRevLett.97.068104
[18] Hrennikov A.: Solution of problems of elasticity by the framework method. Journal of Applied Mechanics, 8, 169-175 (1941).

[19] Kellomäki M., Åström J., Timonen J.: Rigidity and dynamics of random spring networks. Physical Review Letters, 77, 2730-2733 (1996). DOI: $10.1103 /$ PhysRevLett.77.2730

[20] Wilhelm J., Frey E.: Elasticity of stiff polymer networks. Physical Review Letters, 91, 108103/1108103/4 (2003).

DOI: 10.1103/PhysRevLett.91.108103

[21] Head D. A., Levine A. J., MacKintosh F. C.: Deformation of cross-linked semiflexible polymer networks. Physical Review Letters, 91, 108102/1108102/4 (2003).

DOI: 10.1103/PhysRevLett.91.108102

[22] Buxton G. A., Clarke N.: 'Bending to stretching' transition in disordered networks. Physical Review Letters, 98, 238103/1-238103/4 (2007).

DOI: 10.1103/PhysRevLett.98.238103

[23] Broedersz C. P., Storm C., MacKintosh F. C.: Nonlinear elasticity of composite networks of stiff biopolymers with flexible linkers. Physical Review Letters, 101, 118103/1-118103/4 (2008). DOI: 10.1103/PhysRevLett.101.118103

[24] Gibson M. A., Bruck J.: Efficient exact stochastic simulation of chemical systems with many species and many channels. Journal of Physical Chemistry A, 104, 1876-1889 (2000). DOI: $10.1021 / j p 993732 q$

[25] Lodish H., Berk A., Kaiser C. A., Krieger M., Scott M. P., Bretscher A., Ploegh H., Matsudaira P.: Molecular cell biology. Freeman, San Francisco (2007).

[26] Ott A., Magnasco M., Simon A., Libchaber A.: Measurement of the persistence length of polymerized actin using fluorescence microscopy. Physical Review Letters, 48, R1642-R1645 (1993).

[27] Käs J., Strey H., Tang J. X., Finger D., Ezzell R., Sackmann E., Janmey P. A.: F-actin, a model polymer for semiflexible chains in dilute, semidilute, and liquid crystalline solutions. Biophysical Journal, 70, 609625 (1996). DOI: $\underline{10.1016 / \mathrm{S} 0006-3495(96) 79630-3}$

[28] Ostoja-Starzewski M., Stahl D. C.: Random fiber networks and special elastic orthotropy of paper. Journal of Elasticity, 60, 131-149 (2000). DOI: 10.1023/A:1010844929730

[29] Isambert H., Maggs A. C.: Dynamics and rheology of actin solutions. Macromolecules, 29, 1036-1040 (1996).

DOI: $10.1021 / \mathrm{ma} 946418 \mathrm{x}$

[30] Meyer R. K., Aebi U.: Bundling of actin filaments by A-actinin depends on its molecular length. The Journal of Cell Biology, 110, 2013-2024 (1990). DOI: $10.1083 /$ jcb.110.6.2013

[31] Landau L. D., Lifshitz E. M.: Theory of elasticity. Pergamon Press, Oxford (1986). 
[32] MacKintosh F. C., Kas J., Jamney P. A.: Elasticity of semiflexible biopolymer networks. Physical Review Letters, 75, 4425-4428 (1995). DOI: $10.1103 /$ PhysRevLett.75.4425

[33] Gittes F., MacKintosh F. C.: Dynamic shear modulus of a semiflexible polymer network. Physical Review E, 58, R1241-R1244 (1998). DOI: $10.1103 /$ PhysRevE.58.R1241

[34] Liu X., Pollack G. H.: Mechanics of F-actin characterized with microfabricated cantilevers. Biophysical Journal, 83, 2705-2715 (2002). DOI: $10.1016 / \mathrm{S} 0006-3495(02) 75280-6$

[35] Ferrer J. M., Lee H., Chen J., Pelz B., Nakamura F., Kamm R. D., Lang M. J.: Measuring molecular rupture forces between single actin filaments and actinbinding proteins. Proceedings of the National Academy of Sciences, 105, 9221-9226 (2008). DOI: $10.1073 /$ pnas.0706124105

[36] Coffey W. T., Kalmykov Y. P., Waldron J. T.: The Langevin equation. World Scientific, London (1996).

[37] Blanchoin L., Pollard T. D.: Hydrolysis of ATP by polymerized actin depends on the bound divalent cation but not profilin. Biochemistry, 41, 597-602 (2002).

DOI: $10.1021 / \mathrm{bi011214b}$
[38] Fujiwara I., Vavylonis D., Pollard T. D.: Polymerization kinetics of ADP- and ADP-Pi-actin determined by fluorescence microscopy. Proceedings of the National Academy of Sciences, 104, 8827-8832 (2007).

DOI: $10.1073 /$ pnas.0702510104

[39] Gardel M. L., Nakamura F., Hartwig J., Crocker J. C., Strossel T. P., Weitz D. A.: Stress-dependent elasticity of composite actin networks as a model for cell behavior. Physical Review Letters, 96, 088102/1-088102/4 (2006). DOI: 10.1103/PhysRevLett.96.088102

[40] Janmey P. A., Hvidt S., Käs J., Lerche D., Maggs A., Sackmann E., Schliwa M., Stossel T. P.: On the mechanical properties of actin gels. Journal of Biological Chemistry, 269, 32503-32513 (1994).

[41] Gardel M. L., Shin J. H., MacKintosh F. C., Mahadevan L., Matsudaira P., Weitz D. A.: Elastic behavior of cross-linked and bundled actin networks. Science, 304, 1301-1305 (2004).

DOI: $\underline{10.1126 / \text { science. } 1095087}$ 